\title{
Utilização das ferramentas da qualidade nos processos de manutenção, visando o desperdício de tempo e a produtividade
}

\author{
Use of quality tools in maintenance processes, aiming at wasting time and productivity
}

\author{
Lucas S. Penedo*, José Thomaz de Carvalho, Welington L. Costa, Mateus M. E. de Andrade, Pedro \\ L. S. Da Cruz, Thais W. de F. B. Galvão, Tatiane W. de F. B. Galvão
}

Como citar esse artigo. Penedo, LS; De Carvalho, JT; Costa, WL; De Andrade, MME; Da Cruz, PLS; Galvão, TWFB; Galvão, TWFB. Utilização das ferramentas da qualidade nos processos de manutenção, visando o desperdício de tempo e a produtividade. Revista Teccen. 2020 Jan./Jun.; 13 (1): 16-24.

\section{Resumo}

Este artigo, considerando a utilização das ferramentas da qualidade nos processos de manutenção, nos leva a um novo olhar de forma colaborativa, para a reflexão dos gestores da relevância de um sistema de planejamento e controle, visando a produtividade e o desperdício de tempo. Desta forma, este artigo tem como objetivo geral, analisar de que forma ocorre a manutenção de máquinas e equipamentos em empresas da Região Sul Fluminense do estado do Rio de Janeiro, Brasil. Os objetivos específicos são verificar quais ferramentas da qualidade são utilizadas, descrevendo sobre conceitos e a aplicação de cada ferramenta nos processos de manutenção; e analisar os modelos de manutenção existentes nas empresas que fizeram parte da pesquisa, quanto ao controle de equipamentos. A pesquisa de campo de caráter quantitativo, realizada por meio de questionário com 14 questões fechadas, encaminhado à profissionais da área de manutenção, por meio do Google Forms, que é um serviço gratuito, destinado à criação e pesquisas online, que viabilizou a confirmação da hipótese que foi verificar se a implementação de modelos de manutenção por meio de sistemas de planejamento e controle, com a utilização de ferramentas da qualidade, pode contribuir para o desperdício de tempo e com a produtividade. Os resultados obtidos com a pesquisa de campo atenderam aos objetivos propostos e confirmaram a hipótese deste artigo.

Palavras-Chave: Planejamento; Controle; Manutenção; Ferramentas da qualidade.

\begin{abstract}
This article, considering the use of quality tools in maintenance processes, takes us to a new look in a collaborative way, for managers to reflect on the relevance of a planning and control system, aiming at productivity and wasting time. Thus, this article has the general objective of analyzing how the maintenance of machinery and equipment occurs in companies in the Southern Fluminense Region of the state of Rio de Janeiro, Brazil. The specific objectives are to verify which quality tools are used, describing concepts and the application of each tool in the maintenance processes; and to analyze the maintenance models existing in the companies that were part of the research, regarding the control of equipment. The quantitative field research, carried out through a questionnaire with 14 closed questions, forwarded to professionals in the maintenance area, through Google Forms, which is a free service, aimed at creating and researching online, which made it possible to confirm the hypothesis that was to verify if the implementation of maintenance models through planning and control systems, with the use of quality tools, can contribute to the waste of time and productivity. The results obtained with the field research met the proposed objectives and confirmed the hypothesis of this article.

Keywords: Water quality; Water Resources Management; Quality of life.
\end{abstract}

\section{Introdução}

Nos ambientes organizacionais é indispensável a gestão, para a análise e avaliação do processo produtivo e consequentemente, para o alcance das metas e posição favorável no mercado. A redução de custos e a luta contra o desperdício são primordiais para garantir a competitividade, viabilidade do empreendimento e atingir seu objetivo final que é a satisfação dos clientes, mas, isso só é possível com o melhoramento contínuo dos processos.
Atualmente, a gestão da qualidade abrange uma visão sistêmica, influenciando as pessoas no modo de pensar e agir. O significado da qualidade anteriormente entendida como controle da produção e a qualidade intrínseca de bens e serviços, uso de ferramentas, método de gestão e assistência técnica adequada, hoje entendida como a qualidade total ou gestão da qualidade por um modelo de gerenciamento que busca a eficiência e a eficácia, em todas as áreas das organizações. Dentre os benefícios da gestão da qualidade, estão a facilidade do inter-relacionamento e da satisfação do

Afiliação dos autores:

Universidade de Vassouras, UV, Vassouras, RJ, Brasil 
cliente e a capacidade de prover produtos e serviços que atendam aos requisitos do usuário e dos agentes regulamentadores.

Desta forma, considerando que a gestão da qualidade abrange toda infraestrutura organizacional, a manutenção tem adquirido mais confiabilidade, uma vez que equipamentos e maquinários em perfeito estado de funcionamento, garantem continuidade e qualidade no processo produtivo e melhora a eficiência da manutenção, evitando desperdício de investimentos e tempo morto (TM).

\section{Tipos de manutenção}

Os tipos de manutenção são caracterizados pela forma como as intervenções são realizadas nos equipamentos, nos sistemas ou instalações. Neste artigo, serão descritas seis práticas básicas de manutenção, consideradas como principais por diferentes autores. São elas: manutenção corretiva planejada e não-planejada, manutenção preventiva, manutenção preditiva, manutenção detectiva e engenharia de manutenção.

\section{Manutenção corretiva}

A Associação Brasileira de Normas Técnicas (apud, Otani e Machado, 2008), destaca que a manutenção corretiva é o método efetuado após a ocorrência de uma pane destinada a recolocar um item em condições de executar uma função requerida. Segundo Botelho e Bifano (2015) a manutenção corretiva é realizada após ter sido apresentado o defeito, sendo a mais elementar das manutenções, mas, Botelho e Bifano (2015) advertem que seu uso permanente pode gerar paralizações de processos, sem possibilidade de previsão. Seleme (2015) afirma que a manutenção corretiva é efetuada após a ocorrência de uma pane e destinada a recolocar um item em condições de executar uma função requerida. Apesar das definições apontarem para uma manutenção simples e elementar, essa abordagem ainda se subdivide em duas categorias: planejada e não-planejada.

A manutenção corretiva não-planejada é aquela que ocorre quando a correção da falha ou do desempenho abaixo do esperado é realizada sempre após a ocorrência do fato, sem acompanhamento ou planejamento anterior. Implica em altos custos e baixa confiabilidade de produção, já que gera ociosidade e danos maiores aos equipamentos, muitas vezes irreversíveis (Otani \& Machado, 2008).

A manutenção corretiva planejada é aquela que ocorre quando a manutenção é preparada. Otani e Machado (2008) apontam que "pelo seu próprio nome planejado, indica que tudo o que é planejado, tende a ficar mais barato, mais seguro e mais rápido".

\section{Manutenção preventiva}

Seleme (2015) afirma que este tipo de manutenção é efetuado em intervalos predeterminados ou de acordo com critérios prescritos, destinados a reduzir a probabilidade de falhas ou de mal funcionamento de um item. Botelho e Bifano (2015) relatam que a manutenção preventiva se baseia em informações de durabilidade de equipamentos, materiais e instalações. Almeida (2000) destaca que:

\footnotetext{
"todos os programas de gerência de manutenção preventiva assumem que as máquinas degradarão com um quadro típico de sua classificação em particular, ou seja, os reparos e recondicionamentos de máquinas, na maioria das empresas, são planejados a partir de estatísticas, sendo a mais largamente usada a curva do tempo médio para falha - CTMF."
}

\section{Manutenção preditiva}

Nesse tipo de manutenção se acompanha o controle de tolerâncias, medição de preditivas, como por exemplo disjuntores e vibrações anormais entre outros, necessitando de levantamentos estatísticos confiáveis. A vantagem desse sistema de manutenção é que os equipamentos não chegam a ser danificados e os períodos de verificação podem ser aumentados (Botelho \& Bofani, 2015).

Segundo Almeida (2000) a manutenção preditiva "é um meio para melhorar a produtividade, a qualidade do produto, o lucro, e a efetividade global das plantas industriais de manufatura e de produção". A Associação Brasileira de Normas Técnicas (apud, Pereira, 2011), afirma que a manutenção preditiva permite garantir a qualidade de serviço desejada, com base na aplicação sistemática de técnicas de análise, utilizando-se meios de supervisão centralizados ou de amostragem. Seleme (2015) ressalta que, a manutenção preditiva permite garantir uma qualidade de serviço desejada, com base na aplicação sistemática de técnicas de análise, utilizandose de meios de supervisão centralizados.

\section{Manutenção detectiva}

A manutenção detectiva (detectar) é evidenciada a partir da década de 90. Souza (2008) destaca que o objetivo desse tipo de manutenção é aumentar a confiabilidade dos equipamentos, considerando que é caracterizada pela intervenção em sistemas de proteção para detectar falhas ocultas e não perceptíveis ao pessoal da operação.

\section{Engenharia de manutenção}

Engenharia de manutenção é o ramo da engenharia 
responsável pelo planejamento, execução e controle das atividades de manutenção, focado na identificação e análise das falhas, de modo que elas não tornem a acontecer.

Segundo Seleme (2015):

“...engenharia de manutenção é uma atividade de manutenção de equipamentos/itens que pressupões $o$ desenvolvimento de conceitos, critérios e requisitos técnicos nas fases conceituais e de aquisição, devendo o resultado ser utilizado e mantido durante a fase operacional, assegurando o apoio à manutenção eficaz dos equipamentos"

Para Kardec e Nascif (2012) a prática da engenharia de manutenção significa uma mudança cultural, uma quebra de paradigma, através da consolidação de rotinas de trabalho e da implantação de melhorias. A empresa que pratica a engenharia de manutenção,nãoestáapenasrealizandoacompanhamento preditivo de seus equipamentos e máquinas, ela está alimentando sua estrutura de dados e informações sobre manutenção que irão lhe permitir realizar análises e estudos, para proposição de melhorias no futuro. A Figura 01 ilustra melhor as diferenças entre os diversos tipos de manutenção e a posição da engenharia de manutenção que interage com todas, neste cenário.

\section{Qualidade na manutenção}

Um bom gerenciamento consiste em saber localizar problemas e então planejar a melhor maneira de resolvê-los. Os maiores benefícios da qualidade na manutenção, se resumem em aumento da produtividade e permanência do nível funcional dos equipamentos e maquinários. Nesse contexto, as ferramentas e metodologias da qualidade estão cada vez mais presentes nos processos de gestão da manutenção. Considerase na metodologia, o método de análise de solução de problemas (MASP) que, segundo Hilgemberg (2015) é um procedimento para elaboração de relatórios, por meio de pessoas e reportam os resultados de melhorias, obtidas no âmbito da qualidade. A partir desses relatórios surgiu o nome pelo qual esse método é mundialmente conhecido, QC Story (Sugiura \& Yamada, 1995 apud Hilgemberg, 2015).

O MASP tornou-se um método muito utilizado na solução de problemas devido a sua facilidade de uso e entendimento. Este método aumenta a eficiência de melhoria da qualidade, além de estar em paralelo com a ferramenta de controle e gerenciamento de processos, que é o ciclo plan-do-check-act (PDCA).

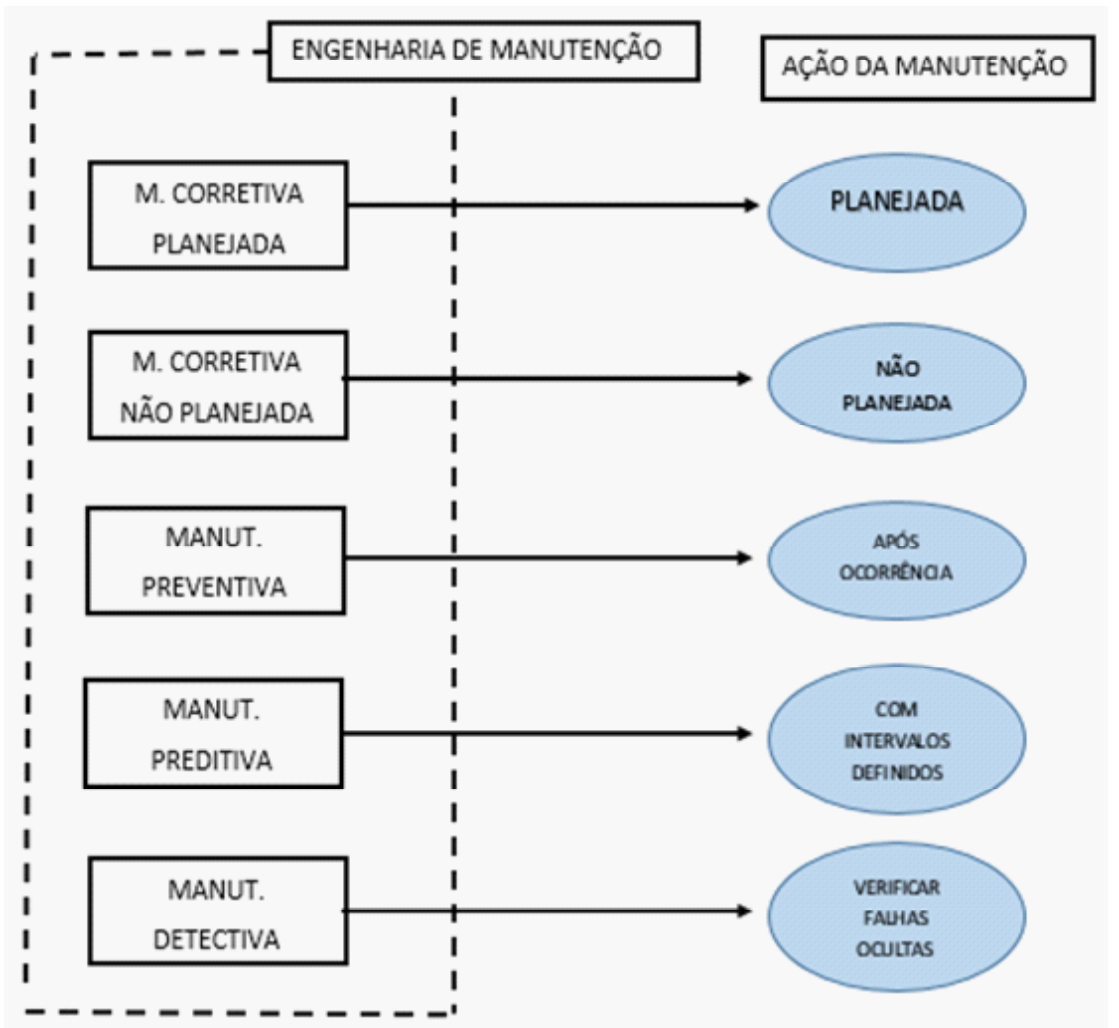

Figura 1. diferenças entre os tipos de manutenção e a posição da engenharia de manutenção. 


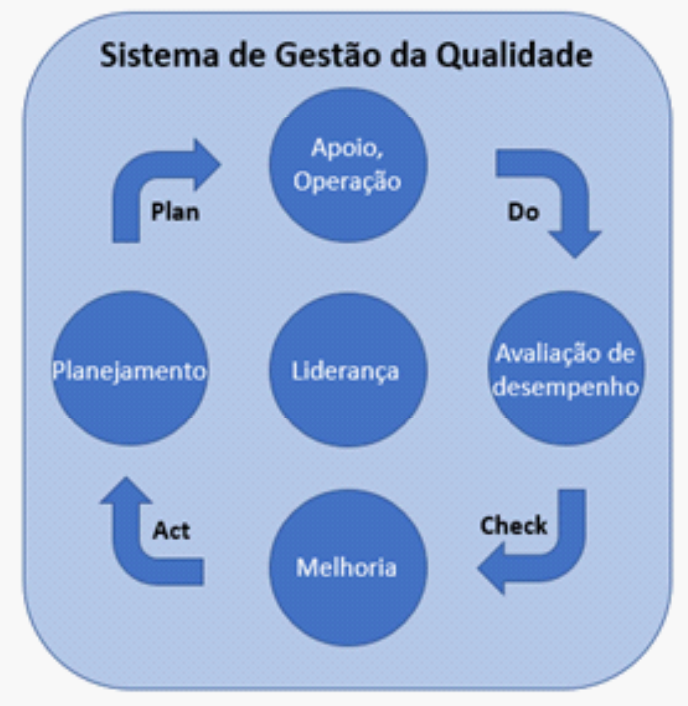

Figura 2. Representação do ciclo PDCA.

Fonte: Autores, 2020

\section{Ciclo plan-do-check-act}

O ciclo plan-do-Check-act (PDCA), representado na Figura 02, é um método de gerenciamento que tem por objetivo a manutenção e a melhoria dos processos, considerando que em cada etapa de sua aplicação são utilizadas diversas ferramentas da qualidade (Junior et al., 2008).

De acordo com a NBR ISO 9001-2015 esse método pode ser aplicado em todo e qualquer processo. A metodologia consiste em promoção da melhoria contínua, por meio de quatro etapas integradas:

a) Plan (planejar): traçar objetivos do processo bem como recursos necessários para a realização dele, visando atender as expectativas do cliente;

b) Do (fazer): colocar em pratica o planejado;

c) Check (checar): acompanhar e realizar medições dos processos, produtos e serviços e analisar resultados e;

d) Act (agir): realizar ações de melhoria no desempenho do processo.

Ainda segundo a NBR ISO 9001-2015 a implantação do ciclo PDCA na manutenção com as ferramentas da qualidade, tem como objetivo a maior redução possível de falha, defeito e perda, além de maior disponibilidade dos equipamentos e confiabilidade nos processos, sem desperdício de tempo. A aplicação do PDCA na gestão da manutenção é extremamente eficaz para garantir a melhoria contínua dos processos de manutenção.

\section{Ferramentas da qualidade}

Para auxiliar no dia a dia com as ocorrências de manutenção, falhas de equipamentos e maquinários, nos ambientes organizacionais existem ferramentas e metodologias que podem ser adotadas, facilitando assim o direcionamento de nossas decisões, desperdício de tempo e o foco que realmente impacta na produtividade e nos resultados da empresa.

Por meio da apresentação de conceitos das ferramentas é possível perceber e entender melhor a relevância das mesmas. Neste sentido se faz necessário, o conhecimento da aplicação das ferramentas da qualidade, conhecidas também por: ferramentas de gerenciamento e ou ferramentas estatísticas para o controle de qualidade que são apresentados a seguir:

a) Carta de controle: por mais controlado que um processo seja, ele sempre sofrerá algum tipo de variação. Controlar essa variação é fundamental para garantir a qualidade de bens e serviços. A carta ou gráfico de controle é a ferramenta que permite a análise da variação à qual um processo está submetido, mostrando se essa variação está dentro do padrão médio esperado ou se apresenta um desvio que precisa ser investigado. Segundo Junior et al. (2008) carta de controle é um tipo de gráfico que serve para acompanhar a variabilidade de um processo, identificando suas causas comuns (intrínsecas ao processo) e especiais (aleatórias).

b) Diagrama de causa e efeito: também conhecido como de diagrama de Ishikawa ou espinha de peixe (por causa de seu formato), o diagrama de causa e efeito é aquele que agrupa as causas por categorias e semelhanças previamente estabelecidas, ou percebidas durante o processo de classificação (Junior et al., 2008).

c) Fluxograma: é uma representação gráfica que permite a fácil visualização dos passos de um processo. O Fluxograma serve para descrever o processo, mas, é preciso conhecer o processo para controlá-lo por meio desta ferramenta. Neste sentido, Brasil (2011) afirma que sua maior vantagem é propiciar visão completa do processo e delimitar cada uma de suas etapas.

d) Folha de verificação: ferramenta usada para quantificar a frequência com que certos eventos ocorrem num determinado período de tempo (Junior et al. 2008).

e) Histograma: é um gráfico de barras que mostra a distribuição de dados por categorias. Conforme Junior et al. (2008), enquanto os gráficos de controle mostram o comportamento de uma variável ao longo do tempo, o histograma fornece uma fotografia variável num determinado instante. Relatam também que as frequências são agrupadas estatisticamente na forma de classes, nas quais se observa a tendência central dos valores e sua variabilidade.

f) Diagrama ou Gráfico de Pareto: trata-se de um 
gráfico de barras, construído a partir de um processo de coleta de dados (em geral, uma folha de verificação), podendo ser usado quando se deseja priorizar problemas ou causas relativas a um determinado assunto. Segundo Junior et al. (2008), a ideia básica, surgiu a partir do princípio de Pareto (Vilfredo Pareto, economista italiano do século XIX) que foi desenvolvido com base no estudo sobre a desigualdade na distribuição de riquezas, cuja conclusão era de que $20 \%$ da população detinham $80 \%$ das riquezas, enquanto que o restante da população detinha apenas $20 \%$. Relação conhecida como a regra do $80 / 20$.

g) $5 \mathrm{~W} 2 \mathrm{H}$ : ferramenta usada principalmente para o mapeamento e padronização de processos, na elaboração de planos de ação e no estabelecimento de procedimentos associados a indicadores. De acordo com Junior et al. (2008), 5W2H representa as iniciais de palavras, em inglês, sendo 5 (cinco) delas iniciadas por "W" e 2 (duas) delas iniciadas por " $H$ ": why (por que), what (o que), where (onde), when (quando), who (quem), how (como) e how much (quanto custa). Surgiu no mercado uma variação dessa ferramenta, que passou a se chamar $5 \mathrm{~W} 3 \mathrm{H}$, correspondendo o terceiro $\mathrm{H}$ a how many (quantos).

\section{Discussões}

As questões da pesquisa de campo foram relevantes para justificar $\mathrm{o}$ artigo. $\mathrm{O}$ questionário contendo 14 questões com respostas em forma de múltiplas escolhas, sendo 06 questões sobre informações socioprofissionais, foi respondido por 18 profissionais de manutenção, representando $94 \%$ do sexo masculino e $6 \%$ do sexo feminino. A idade dos participantes da pesquisa foi entre 22 anos e 59 anos. A formação educacional dos respondentes da pesquisa apresentou resultado excelente: $61 \%$ com formação no ensino superior e 56\% com formação técnica, mostrando que o interesse pela "práxis" está em coerência com o mercado de trabalho.

Quanto ao ramo de negócio da empresa em que trabalha, a pesquisa observou nas respostas, a diversificação de empresas de manutenção da Região Sul Fluminense, em diferentes ramos, tais como: 4 concessionárias de energia, 1 metalúrgica, 1 siderúrgica, 3 empresas de telecomunicações, 2 mecânicas, 2 ferroviárias, 1 subestação blindada de média tensão e 4 distribuidoras de energia.

Quanto ao cargo e ou função, a maioria dos respondentes da pesquisa estão trabalhando com "manutenção", direta ou indiretamente. $49 \%$ trabalhando como eletricista de manutenção, $22 \%$ trabalhando como técnico em eletrônica, $21 \%$ como instrutor técnico e $8 \%$ como assistente de planejamento e controle de manutenção (PCM). Quanto ao tempo de serviço dos colaboradores nas empresas, ficou entre 2 a 8 anos.

Dentre os modelos de manutenção apresentados no questionário, os respondentes destacaram os mais utilizados pela empresa, conforme demonstrado no Gráfico 01. Observa-se que a manutenção preventiva e a manutenção corretiva não planejada, representando $32 \%$ e $27 \%$ respectivamente, são as mais utilizadas pelas empresas da pesquisa. Considerando esses percentuais, pode-se afirmar que as empresas pesquisadas, utilizam esses métodos de manutenção, com foco na redução de falhas ou de mal funcionamento de um equipamento, sempre após a ocorrência do fato, o que pode ocasionar em altos custos, baixa produtividade gerando ociosidade e problemas nos equipamentos que muitas vezes são irreversíveis.

Quanto a maneira em que os setores de manutenção das empresas são organizados, $50 \%$ dos respondentes apontaram ser descentralizado e $22,2 \%$ afirmaram ser centralizado, conforme demonstrado no Gráfico 02.

Quanto a utilização de ferramentas da qualidade

Gráfico 1. Modelos de manutenção utilizados nas empresas.

\title{
MODELOS DE MANUTENÇÃO UTILIZADOS PELAS
} EMPRESAS

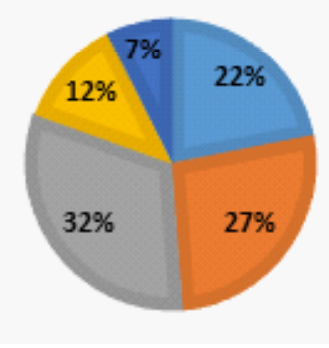

\author{
- Manut. Corretiva Planejada \\ - Manut. Corretiva não Planejada \\ manut. Preventiva \\ - Manut. Preditiva \\ Manut. Detectiva \\ - Não sei informar
}


Gráfico 2. Forma em que os setores de manutenção são organizados.

\section{O setor de manutenção, em sua empresa é organizado de que modo?} 18 respostas

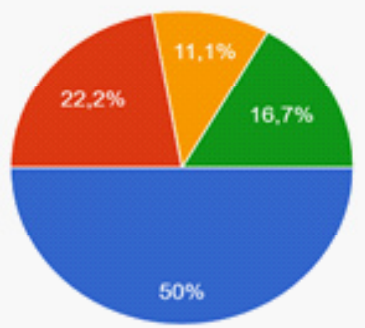

Descentralizado (Existe uma equipe de manutençâo para cada processo)

- Centralizado (Existe apenas uma equipe de manutençâo que atua em toda empresa)

Misto

Manutençào Terceirizada

Nào sei informar

e por meio de quais ferramentas a manutenção é realizada nas empresas, os respondentes afirmaram que o diagrama de causa e efeito, a folha de verificação, o fluxograma e o histograma, representando 38,9\%, 33,3\% e $27,8 \%$, são as ferramentas mais utilizadas no setor de manutenção das empresas. Percebe-se que o diagrama de causa e efeito é a ferramenta mais representativa nas empresas pesquisadas. Esta ferramenta permite detectar as possíveis causas e soluções do problema, envolvendo toda a equipe de manutenção, objetivando a eliminação do problema com mais eficiência e eficácia. Quanto a folha de ocorrência, permite uma análise relacionada a frequência do fato. O fluxograma, demonstra passo a passo, a sequência do trabalho e o histograma, a representação gráfica construído com resultados das ocorrências. Considera-se por meio dos resultados apresentados, que as empresas pesquisadas utilizam as ferramentas adequadas aos seus tipos de produtos e equipamentos. Conforme representado no Gráfico 03.

Quanto ao diagnóstico das causas das principais falhas e se a empresa possui ferramentas para análise das mesmas, bem como, se mantém histórico das causas das falhas, conforme demonstrado no Gráfico 04, os respondentes destacaram que $66,7 \%$ das causas e falhas, são diagnosticadas e registradas por meio de ferramentas de análise de falhas.

Quanto a avaliação e monitoria dos riscos das falhas de maneira a permitir o controle dos equipamentos, como se pode observar no Gráfico 05, $44,4 \%$ dos respondentes afirmaram que, a empresa avalia e monitora os riscos das falhas em todas as áreas e 33,3\% relataram que sim, apenas em equipamentos e ou setores críticos.

Buscando saber se a empresa utiliza algum sistema manual ou informatizado, de planejamento e controle de manutenção, 50\% dos respondentes relataram que sim, é informatizado, planeja, controla e gerencia todos os serviços ligados à manutenção, conforme demonstrado no Gráfico 06.

Ao responderem sobre a questão se o setor de manutenção atende as expectativas e a demanda da empresa, 55,6\% dos respondentes afirmaram que sim,

Gráfico 3. Forma em que os setores de manutenção são organizados.

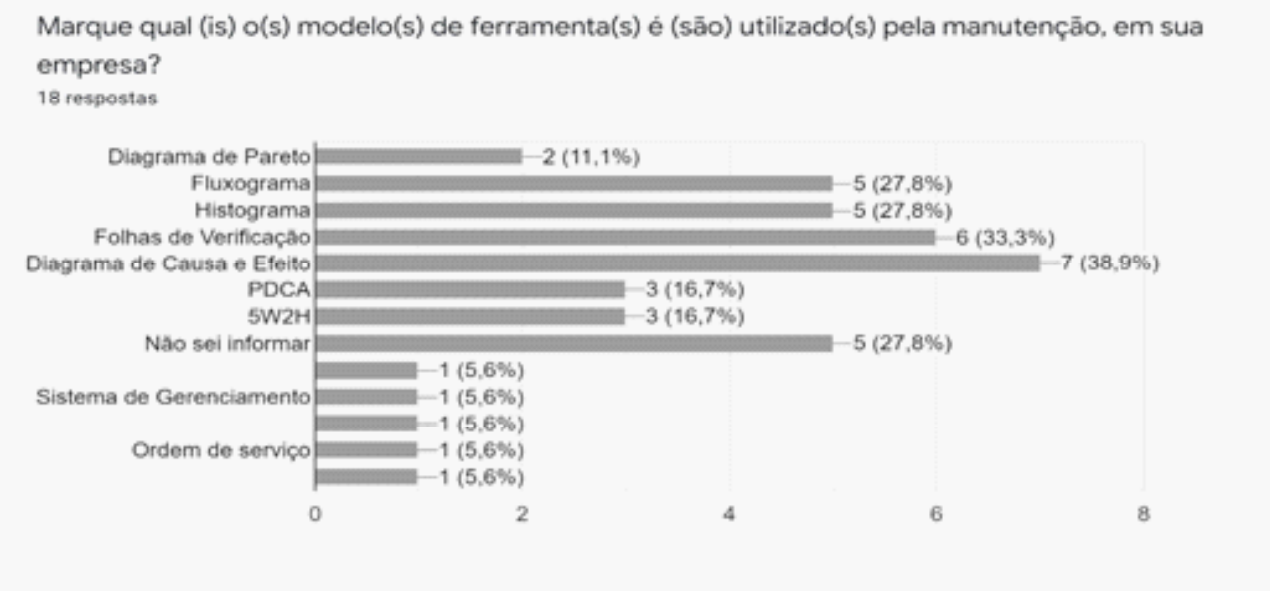


Gráfico 4. Diagnóstico, análise e histórico das principais causas das falhas.

A empresa busca diagnosticar a causa das principais falhas e possui ferramentas para análise das mesmas, bem como mantém um histórico das causas das falhas?

18 respostas

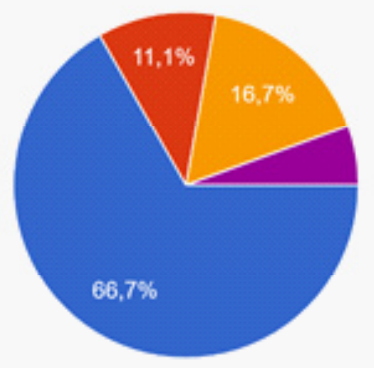

Sim, as falhas são diagnosticadas e registradas por meio de ferramentas $\mathrm{d}$...

- Sim, as falhas são diagnosticadas mas. năo há registros nem histórico das fal...

Sim. mas, algumas vezes não se consegue diagnosticar e as falhas săo..

- Não, o diagnóstico e análise de falhas...

- Não há diagnostico nem registro

Opçáo 6

- Não sei informar

Gráfico 5. Avaliação e monitoração das falhas visando permitir controle de equipamentos.

A empresa avalia e monitora os riscos das falhas de maneira a permitir o controle dos equipamentos?

18 respostas

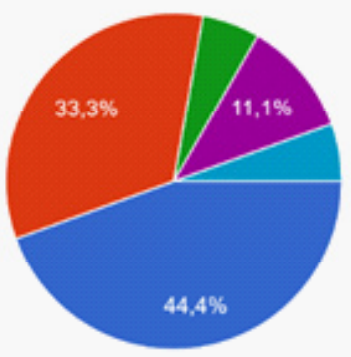

Sim, em todas as áreas

Sim, apenas em equipamentos e ou setores criticos

Sim, apenas quando os riscos envolvem a segurança e o meio ambiente

Sim, mas, năo é eficaz

Nâo avalia nem monitora os riscos das falhas

Nẫ sei informar

Fonte: Autores, 2020

Gráfico 6. Resultados relativos à empresa possuir sistema manual e ou informatizado de planejamento e controle de manutenção.

Sua empresa utiliza algum sistema informatizado ou manual, de Planejamento e Controle de manutenção?

18 respostas

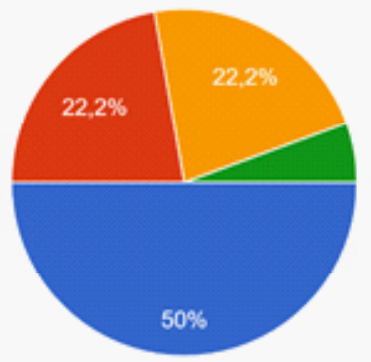

Sim, é informatizado e planeja, controla e gerencia todos os serviços ligados à manutençåo

Sim é informatizado e planeja, controla e gerencla todos os serviços ligados à manutenção, mas, há falhas na priorizaçăo ou alocaçăo de recursos

- Não existe controle relativo ao setor de manutençâo

Não sei informar 
na maioria das vezes os serviços de manutenção são atendidos no tempo esperado. Conforme representado no Gráfico 07.

Quanto a consideração dos custos de operação ao realizar um investimento em novos equipamentos, $61,1 \%$ dos respondentes afirmaram que sim, a maioria dos investimentos. Conforme demonstrado no Gráfico 08.

\section{Conclusão}

Por meio da pesquisa de campo deste artigo, foi possível identificar que os modelos de manutenção mais utilizados nas empresas são a manutenção preventiva e a manutenção corretiva não planejada. Neste sentido, observa-se que existe planejamento para os demais modelos de manutenção, exceto a corretiva, em que, após ocorrência é que se executa a manutenção.

As ferramentas apontadas como as mais utilizadas pela manutenção nas empresas foram: diagrama de causa e efeito e folhas de verificação. Essas ferramentas são relevantes para solucionar problemas de forma eficaz. Verificou-se na pesquisa que os profissionais de manutenção buscam diagnosticar e analisar as causas das falhas, registrando-as, bem como, avaliando e monitorando permitindo controle de seus equipamentos.

Percebeu-se com os resultados da pesquisa que $50 \%$ das empresas possuem sistemas de planejamento e controle de manutenção, informatizado (incluindo o PDCA). Os custos de operação de manutenção com relação aos investimentos de novos equipamentos, também são controlados. Com os resultados apresentados, observa-se que os gestores dos setores

Gráfico 7. O setor de manutenção atende as expectativas da empresa.

\section{O setor de manutenção atende as expectativas e a demanda da empresa? \\ 18 respostas}

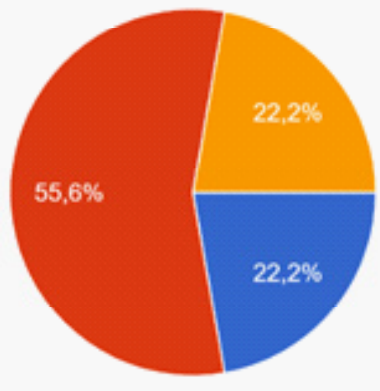

Sim, os serviços de manutenção sâo sempre atendidos no tempo esperado

Sim, na maioria das vezes os serviços de manutençăo săo atendidos no tempo esperado

Sim, mas, por vezes os serviços de manutenção sofrem com atrasos $\mathrm{e}$ ou com má qualidade.

Não atende

Não sei informar

Gráfico 8. Consideração dos custos de operação ao realizar novos investimentos.

Ao realizar um investimento em novos equipamentos são considerados também os custos de operação de manutenção?

18 respostas

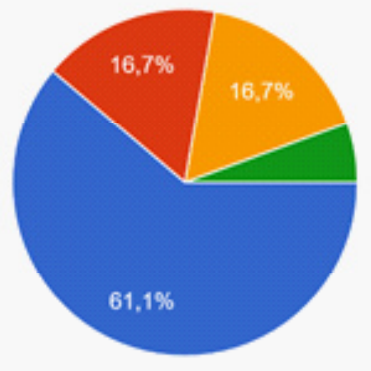

Sim, na maioria dos investimentos

Sim, apenas nos investimentos altos

Não

Não sei informas 
de manutenção estão focados na melhoria contínua, no desperdício de tempo e na produtividade.

Os itens que merecem maior atenção dos gestores na manutenção são os seguintes: planejamento da manutenção; maior utilização de fluxogramas e mapeamento de processos; e qualidade na manutenção dos equipamentos.

Com a metodologia utilizada nesta pesquisa foi possível obter dados para análise, de diferentes profissionais envolvidos na manutenção que, tiveram a oportunidade de registrar suas percepções sobre a manutenção que gerenciam ou executam nas organizações em que trabalham.

Os resultados da pesquisa atenderam aos objetivos propostos e a confirmação da hipótese deste artigo, ao perceber que, a aplicação de modelos de manutenção e ferramentas da qualidade podem contribuir para a produtividade sem desperdício de tempo. Sugere-se uma pesquisa mais aprofundada sobre a viabilidade técnica, de implantação de um setor de engenharia de manutenção nas empresas pesquisadas.

\section{Referências}

Almeida, M. T. (2000). Manutenção Preditiva: Confiabilidade e Qualidade.

Botelho, M.H.C., Bifano, M.H.(2015). Operação deCaldeiras: gerenciamento, controle e manutenção. São Paulo: Bleucher.

Brasil, Pearson Education do. (2011). Gestão da Qualidade. São Paulo: Pearson.

Costa, M. A. (2013). Gestão Estratégia da Manutenção: uma oportunidade para melhora o resultado operacional. Trabalho de Conclusão de Curso (Graduação em Engenharia de Produção). Juiz de Fora: UFJF.

Hilgemberg, Daniel. (2015). Redução de variação de tonalidade e defeitos superficiais em placas cerâmicas utilizando o método de análise e solução de problemas (MASP).

Junior, I. M., Cierco A. A., Rocha, A. V., Mota, E. B. Leusin, S. (2008). Gestão da Qualidade. 9. Ed. Rio de janeiro: FGV:

Kardec, Alan; Nascif, Júlio. (2012) Manutenção - Função Estratégica. Rio de Janeiro: Qualitymark Editora.

NBR ISO 9001 (2015) - ABNT ISO 9001 (2015). Sistema de Gestão da Qualidade. São Paulo: Requisitos.

Otani, M.; Machado, W. V. (2008). A proposta de desenvolvimento de gestão da manutenção industrial na busca da excelência ou classe mundial.

Revista Gestão Industrial. Vol.4, n.2.

Pereira, Mário Jorge. (2011). Engenharia de Manutenção - Teoria e Prática. 1. ed. Rio de Janeiro: Editora Ciência Moderna.

Seleme, Robson. (2015). Manutenção Industrial: mantendo a fábrica em funcionamento. Curitiba: InterSaberes.

Souza, J. B. (2008). Alinhamento das estratégias do Planejamento e Controle da Manutenção (PCM) com as finalidades e função do Planejamento e Controle da Produção (PCP): Uma abordagem Analítica. 169f. Dissertação (Mestrado em Engenharia de Produção). Paraná: UTFP.

Viana, Herbert Ricardo Garcia. (2002). PCM - Planejamento e Controle de Manutenção. Rio de Janeiro: Qualitymark Editora. 